\title{
Stereoscopic perception with brief exposures
}

\author{
WILLIAM R. UTTAL \\ Arizona State University, Tempe, Arizona \\ and \\ NANCY S. DAVIS and CYNTHIA WELKE \\ SEACO, Kailua, Hawaii
}

\begin{abstract}
In this report we describe the results of an experiment in which we demonstrated that a powerful and compelling stereoscopic experience is elicited with very brief $(<1 \mathrm{msec})$ stimulus durations. The observers were highly successful in recognizing briefly flashed, stereoscopic, random-dot surfaces in the absence of monocular contours. The results are shown to be closely related to the range of depths for any stimulus form; however, the recognition thresholds were nonmonotonic as a function of disparity. Previous investigators have disagreed about the existence of a temporal threshold for stereopsis. We believe that prior findings suggesting that stereopsis cannot occur at short exposure durations are probably due to inadequate control of fixation disparity. Therefore, there is poor dichoptic image registration when a stereoscopic stimulus is presented. The present results also raise difficulties for any theory of stereopsis that requires eye movements.
\end{abstract}

How long must a stimulus last for stereoscopic perception to occur when there are no monocular cues? There is some dispute over this question in the literature. Lehmkuhle and Fox (1980), who studied the effect of stereoscopic depth on metacontrast masking (produced by random-dot stereograms), demonstrated perceived depths at stimulus durations as low as $50 \mathrm{msec}$. On the other hand, Richards (1977) reported that depth in briefly presented random-dot stereograms cannot be perceived (unless monocular cues are present) when stimulus durations are less than $200 \mathrm{msec}$. Richards asserted that eye movements are necessary for many subjects to see random-dot stereograms.

In this paper, we demonstrate that brevity of exposure is not an obstacle to high-quality stereoscopic depth perception even when there are no monocular cues, provided that the dichoptic display is properly registered on corresponding retinal points at the moment the dichoptic stimulus is presented. That is, the retinal disparity at the fixation point should be close to zero, and the line of sight should not have to be adjusted to achieve zero fixation disparity. We proved this point by presenting a dichoptic, random-dot stereogram for a total duration of less than $1 \mathrm{msec}$ in an experimental situation in which subjects' eyes were appropriately preconverged. In pilot studies, it was shown that stimuli of this kind did not lead to any measurable stereoscopic shape recognition if the eyes were not converged at the necessary small fixation

This project was supported by the Office of Naval Research under Work Request N0001485WR24281 and by the Air Force Office of Scientific Research by Grant F49620-92-J-0176P00002. Address correspondence to W. R. Uttal, Perception Laboratory, Department of IMSE, Arizona State University, Tempe AZ 85287. disparity. However, as we show in the present experiment, if care is taken to establish appropriate fixation, even these briefest of practical stimuli can produce performance that approaches $100 \%$ in some conditions.

Our work is closely related to studies (e.g., Patterson \& Fox, 1984b; Steinman \& Collewijn, 1980; Steinman, Levinson, Collewijn, \& van der Steen, 1983) that have shown that excellent stereopsis can be obtained when a subject's head is moving. The usual interpretation of these data is that stereopsis can occur even when strict correspondence is incomplete. This interpretation, in its raw form, would be a counterargument to the one presented in the present paper - that with good correspondence, even briefly presented stimuli can produce good stereopsis.

However, "good" correspondence is a relative term, and the "moving head" findings are not necessarily in disagreement with our present conclusions. Obviously, if the fixation disparities of the eyes were sufficiently disrupted by head motion, then stereoscopic vision would be impossible. Any recorded depth perception must be provided by some other cue-for example, motion parallax. Similarly, in the type of experiment reported here, "good" correspondence does not mean perfect correspondence. In a recent experiment (Uttal, Baruch, \& Allen, 1994), it was demonstrated that "good" correspondence may vary enough so that the effect of random visual dots used as interference may be doubled in dichoptic viewing situations versus binocular or monocular viewing situations. That is, the same number of dots may have twice the masking effect when viewed dichoptically because of slight misregistrations or miscorrespondence between the two eyes. However, even with the slight misregistration that permits the small dots to be effectively doubled in their masking effect, 
stereoscopic depth may obtain. This is the criterion used in the classic definition of Panum's fusional areas.

We are concerned, therefore, with the entire concept of stereoanomaly. Researchers who have reported that some people may not see stereoscopic depth (Jones, 1977; Richards, 1970, 1971) have been challenged by those using other kinds of experimental techniques. Researchers who have used prolonged viewing (Newhouse \& Uttal, 1982; Patterson \& Fox, 1984a), afterimages (Patterson \& Fox, 1984a), or extensive training (Foley \& Richards, 1974) suggest that, given two eyes and no unusual condition of phoria, "stereoanomaly" may be more appropriately attributed to procedural artifact than to a biological condition.

The results of the present study show that excellent stereopsis can occur with ultrabrief exposures. Thus, they add support to the argument that if the one necessary condition for stereopsis-good fixation disparityobtains, the process is ubiquitous and robust.

\section{METHOD}

\section{Observers}

Four volunteer observers were used. All had normal or corrected vision. As we will show, even under the extreme temporal conditions of this experiment, performance could be classified as either excellent or fair for the 2 male and 2 female subjects. One male and 1 female fell into each of these two performance categories.

\section{Apparatus and Stimuli}

Displays were generated, responses acquired, and the outcomes analyzed automatically with a computer-controlled system. The stimuli consisted of random-dot stereograms of planar, quadric, and cubic three-dimensional (3-D), single-valued surfaces that were similar to those previously used by Julesz (1960) in his pioneering studies of cyclopean vision. The stimulus surfaces were generated in the form of dichoptic stereograms on the face of a Hewlett-Packard 1311-B point-plotting oscilloscope with an ultrafast decay (P-24) phosphor, normally used for flying-spot scanners. The technical manual for this display states that the signal trace would diminish in luminance to $10 \%$ of its original value

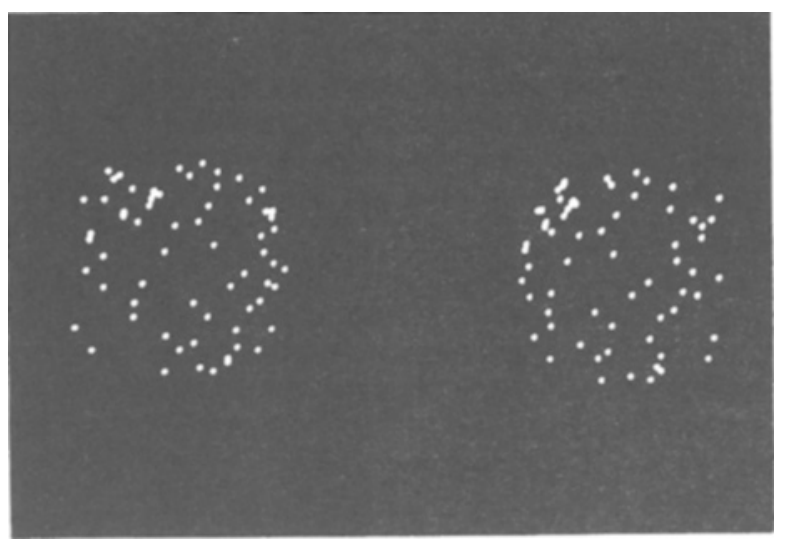

Figure 1. A sample random-dot stereogram, used as a stimulus in the present study. This particular sample is the paraboloid of rotation shown in Figure 2.
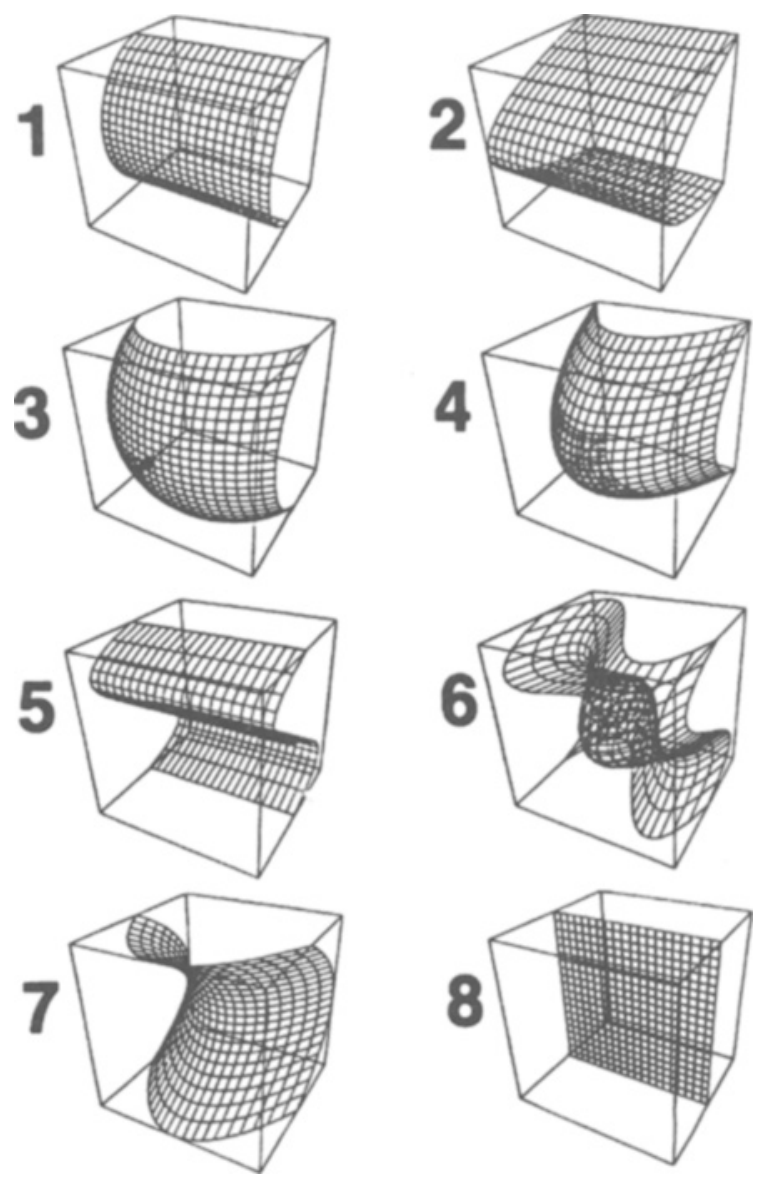

Figure 2. The eight stimulus forms used in the present study. As indicated in the text, these diagrams give a general idea of the shape of the stimuli, but they are not exact. Furthermore, the grid lines and the outline cubes were not present in the actual stimuli; only a collection of dots that varied in their disparity was present. 1 , cylinder; 2, parabolic arch; 3, hemisphere; 4 , paraboloid of rotation; 5 , onedimensional cubic; 6 , two-dimensional cubic ; 7 , hyperbolic paraboloid, or saddle; 8 , plane.

after $1.5 \mu \mathrm{sec}$. The total physical duration of the entire stimulus was certainly less than $1 \mathrm{msec}$. A recent study (Groner, Groner, Muller, Bischof, \& Di Lollo, 1993) has confirmed the brief, effective physical duration of a $\mathrm{P}-15$ phosphor. The $\mathrm{P}-15$ phosphor, which is comparable ( $2.8 \mathrm{vs}$. $1.5 \mu \mathrm{sec})$ to the short-persistence $\mathrm{P}-24$ material used in the present experiment, produced no psychophysically measurable persistence after $1 \mathrm{msec}$.

The observers viewed the screen from a distance of $71 \mathrm{~cm}$. Each image of the stereoscopic pair on the face of the oscilloscope screen subtended a visual angle of $5.0^{\circ} \times 5.0^{\circ}$. Rotary prisms were placed in front of each eye and were individually adjusted by each observer to allow comfortable ocular convergence. The depth dimension of the cube was defined by the dichoptic disparity of the left-eye and right-eye images, as specified in the computer program. Disparity was initially adjusted by the experimenter so that the volume enclosed was judged to be cubical. The disparity limits, thus arbitrarily defined, were subsequently determined to range from $14^{\prime}$ of crossed disparity to $14^{\prime}$ of uncrossed disparity, with fixation (zero disparity) always placed at the center of the viewing cube. Figure 1 shows the appearance of one of the 
sparsely sampled dichoptic stimuli. If viewed through a stereoscope, this stereogram will appear as a paraboloid of rotation, convex toward the observer. If viewed by crossing the eyes, it will appear to be concave.

The images generated on the face of the oscilloscope were created from algorithms and stored prototypes within a Cromemco S-3 microcomputer. The digital representations of the 3-D forms in the computer memory were then converted by means of digital-to-ana$\log$ converters into three analog voltage signals representing the $x$, $y$, and $z$ coordinates of a Cartesian three-space. These signals were then fed into a special-purpose analog computer constructed from Optical Electronics, Inc. components. The purpose of this analog computer was to convert the voltages representing the triplet set of numbers encoding the 3-D coordinates of the dot into two pairs of numbers representing the two-dimensional (2-D) coordinates of a disparate pair of dots-the left- and right-eye haploscopic images--that could evoke the observer's perception of a 3-D nonplanar surface. Because of the analog computer, this conversion could be carried out in real time without burdening the digital computer with extensive and repetitive trigonometric calculations.

The stimulus forms consisted of seven dotted, stereoscopic, nonplanar surfaces and one plane. Samples of these eight forms are shown in Figure 2; however, these are not true depictions on two counts. (1) The figures show the general shapes but not the exact depth dimensions, which have varied from experiment to experiment in our research program. (2) There were no grid lines or outline cubes in the actual stimulus presentations; rather, they appeared simply as a group of point-like dots distributed in the viewing space. The additional lines are presented here only as an aid to visualizing the shape of the surfaces in the 2-D projections with which they must be presented here. The dots in the patterns were briefly illuminated points of minimal size (determined by the point-spread function of the display phosphor), plotted against a black background in a darkened room. It would be impractical to designate the precise luminance of the dots, and the nature of the present experiment and the obtained results suggest that luminance did not exert an important influence on the results over wide ranges. The total number of addressable pixels was $4,096 \times 4,096$ on this high-precision display.

The stimuli were presented without any random masking dots, as in much of our previous research (e.g., Uttal, 1985, 1987; Uttal et al., 1994; Uttal, Davis, Welke, \& Kakarala, 1988). The stimulus degradations necessary to challenge the observer's visual system in the present study were, simply, the brevity of the exposure and the paucity of the dots. The voltage pulse illuminating each dot in each pattern was activated for only $3 \mu \mathrm{sec}$, but, as noted previously, the actual duration was determined by the temporal impulse function of the phosphor and was certainly less than a few microseconds at the worst.

All of the surfaces used as stimulus forms were created by the application of a specific polynomial equation (see Equation 1) to a set of randomly selected $x, y$ coordinate pairs corresponding to positions on a plane. All were created from a single square prototype, whose original dimensions were also $5^{\circ} \times 5^{\circ}$. Each stimulus form contained the same 50 dots in all trials. Therefore, without a perceptual appreciation of the relative disparity of the dots in the stimulus form, they could not be distinguished from each other. Monocular views of the eight forms were very similar, except for the slight dichoptic disparities, unless distributed in space by a functional stereoscopic depth-perceiving system.

The polynomial expression that "stretched" the planar prototype into the seven 3-D surfaces is:

$$
\begin{aligned}
z= & G\left(A x^{5}+B x^{4}+C x^{3}+D x^{2}+E x+R y^{5}+S y^{4}\right. \\
& \left.+T y^{3}+U y^{2}+V y+W\right)^{F+H}
\end{aligned}
$$

By arbitrarily selecting the coefficients $(A, B, C, D, E, F, G, H, R$, $S, T, U, V, W)$ in this equation (including, of course, the option to
Table 1

The Polynomial Equations Used to Stretch the Seven Nonplanar Stimulus Forms From the Planar Prototype

\begin{tabular}{ll}
\hline Stimulus Form & \multicolumn{1}{c}{ Generating Equation } \\
\hline Cylinder & $z=2,535-\left(948,676-y^{2}\right)^{5}$ \\
Parabolic arch & $z=1,099+\left(.002 y^{2}\right)$ \\
Hemisphere & $z=3,221-\left(1,890,625-x^{2}-y^{2}\right) .5$ \\
Paraboloid & $z=1,085+.002\left(x^{2}+y^{2}\right)$ \\
1-D cubic & $z=2,048+.0025\left(.001 y^{3}-948.676 y\right)$ \\
2-D cubic & $z=2,048+.001\left[\left(.001 x^{3}-948.676 x\right)\right.$ \\
& $\left.+\left(.001 y^{3}-948.676 y\right)\right]$ \\
& \\
&
\end{tabular}

Plane $z=2,048$

Note-The numbers in these equations are specific to the details of the equipment in our laboratory and are otherwise unmeaningful. The plane $(z=2,048)$ was placed at the depth of fixation.

set each to zero), it is possible to produce a wide variety of surfaces. The seven polynomial expressions for the seven nonplanar forms used in this study are presented in Table 1. The numerical values in this table are not generally meaningful; they are specific to our computer programs and the nature of the digital-to-analog conversion process. The geometric surfaces used as stimuli were all aligned in a frontoparallel plane, with the main axis of convexity apparently protruding toward the observer by appropriate selection of the disparities.

\section{Psychophysical Procedure}

The experiment was carried out in a speech-recognition mode. That is, the observers were asked to orally enunciate the name of one of the eight stimulus forms by using a set of verbal responses that had been taught to them with a set of diagrams similar to those in Figure 2. An Interstate Electronics Corp. VR200 voicerecognition terminal was used for response acquisition. This device must be trained to respond to each speaker's voice; this preliminary procedure lasted approximately $3 \mathrm{~min}$ and occurred at the beginning of each daily experimental session. Because of the moderate level of recognition accuracy provided by the VR200, it was deemed necessary to also have the computer respond through a VOTRAX voice-generation device with the utterance it had acquired. The observer then accepted or rejected the computer's decision by depressing one of the two hand-held pushbuttons. The intertrial timing of the experiment was, therefore, controlled by the pace of the observer's responses.

In this recognition experiment, a single trial began with the 1 -sec presentation of a dichoptic pair of point-like fixation dots centrally placed in the viewing space. This pair of dots was used by the observer to define the zero-disparity fixation point and, thus, firmly established steady fixation and good stereoscopic fusion when the stimuli were presented. The pair of fixation dots was followed by a single stimulus form, whose total physical duration (when measured in terms of the stream of electronic intensifying pulses used to illuminate its 50 dots) was approximately $300 \mu \mathrm{sec}$.

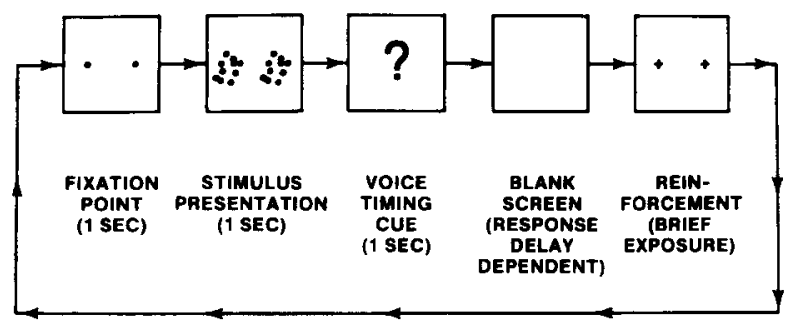

Figure 3. The sequence of events in a single trial of the recognition paradigm used in the study. 
Table 2

Percentage of Total Number of Presentations Correctly Recognized by Observers

\begin{tabular}{ccccc}
\hline & \multicolumn{4}{c}{ Correctly Recognized by Observers } \\
\cline { 2 - 4 } Session & D.S & A.B. & N.D. & N.N. \\
\hline 1 & 62.0 & 90.5 & 85.2 & 40.1 \\
2 & 53.2 & 83.2 & 92.3 & 50.0 \\
3 & 63.3 & 97.3 & 83.8 & 54.3 \\
4 & 61.3 & 92.2 & 97.0 & 61.7 \\
5 & 67.5 & 97.2 & & 62.0 \\
\hline
\end{tabular}

To reiterate, given the short persistence of the P-24 phosphor, the actual physical duration of the stimulus was certainly less than $1 \mathrm{msec}$.

A briefly flashed question mark then concluded the trial sequence. The question mark helped the observer to synchronize his/ her utterance with the acoustic "open window," during which the voice-recognition system was able to accept and process the response. Figure 3 shows the sequence of events in a single experimental trial. In each daily, 1 -h session, the observer was presented with approximately 425 trials, randomly distributed among the eight stimulus forms. The same experiment was run for 5 days for 3 of the observers and for 4 days for 1 .

\section{RESULTS}

The main results of the experiment are shown in Table 2. The performance of each of the observers for each of the 4 or 5 days is tabulated as the percentage of the total number of presentations correctly recognized. There are two important results in this table. The first is that 2 of our observers were, in some sessions (even with these very brief exposures), able to perform at a level $(97 \%)$ that essentially must be considered to be perfect recognition performance (given that a few keying errors and random misjudgments always occur in this type of intensely scheduled psychophysical task). The other 2 observers performed in the $60 \%$ correct range on the average. In spite of the fact that there was some variation among individual observers, clearly there was no limit on stereopsis of a complete and quantitative kind, even at these brief exposures.

The second important result is that the trend in performance levels in this experiment for 3 of our observers showed only modest improvements as a result of practice. This is not, therefore, a task in which there is deep cognitive penetration, nor did the observers require substantial training to learn cryptic cues to the particular forms. The reconstructed awareness of depth, as indicated in their recognition scores, was nearly immediate-not only in terms of the brief exposure, but also in terms of the sequence of the experimental sessions. The process we are studying, therefore, is probably best characterized as mainly low level and preattentive rather than high level and cognitive. This conclusion is also supported by the work of Staller, Lappin, and Fox (1980), which showed that stimulus uncertainty does not impair stereoscopic perception.

Figure 4 displays the effect of stimulus form on the pooled performance scores of all 4 observers. There is obviously a very great discrepancy between the stimulus recognized least frequently (the cylinder-56.58\%) and the one recognized most frequently (the paraboloid of rotation-97.20\%). As is shown in Figure 5, the performance scores for each form are, in large part, predicted by the range of depths over which their constituent dots were distributed. Stimulus forms that were stretched substantially from the prototype plane were almost always recognized in these brief exposures by all 4 observers. The flatter ones that deviated less substantially from the plane were not recognized nearly as often.

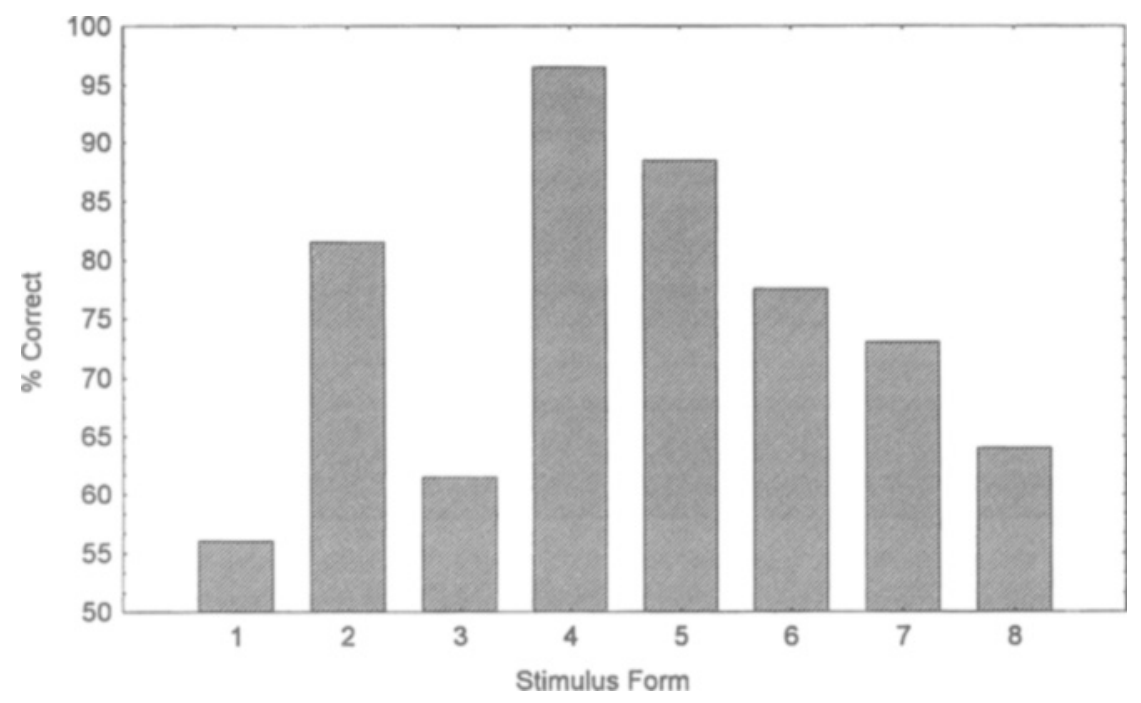

Figure 4. The effect of stimulus form on the recognizability of the stimulus forms utilized in the study. Shaded bars represent percent-correct recognition for cylinder (1); parabolic arch (2); hemisphere (3); paraboloid of rotation (4); one-dimensional cubic (5); two-dimensional cubic (6); hyperbolic paraboloid, or saddle (7); and plane (8). 


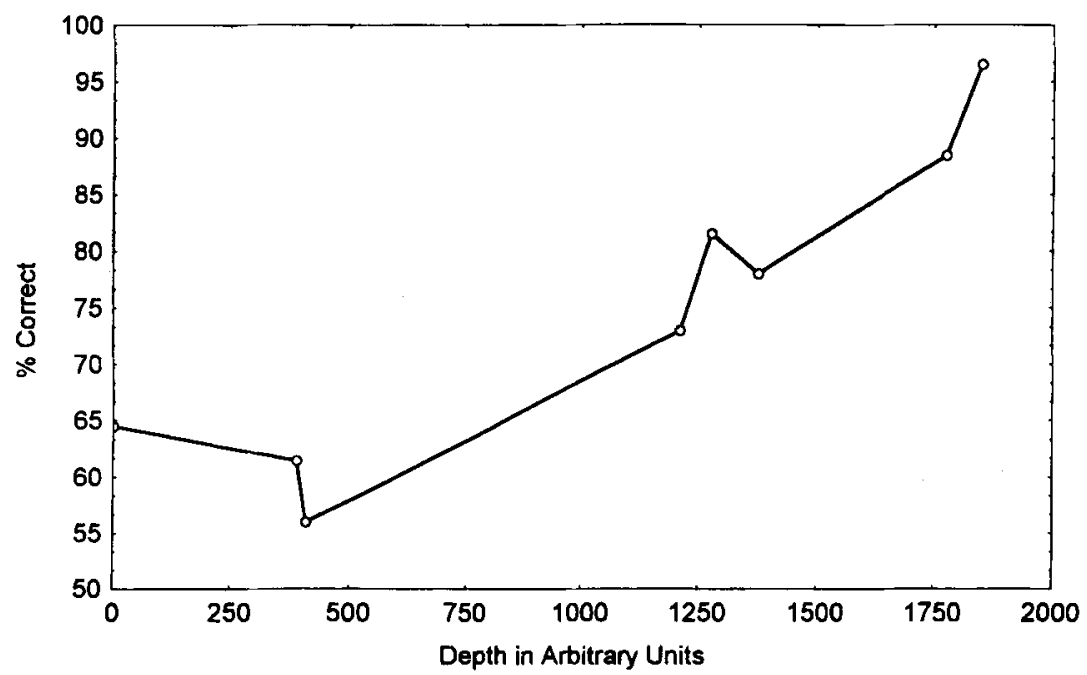

Figure 5. The relationship between the range of depths in any of the stimulus forms and the pooled performance of all the observers in all sessions. The scores in this figure may be crosschecked with those in Figure 4 to identify each shape. The depth is measured in the arbitrary units of Table 1.

A Cox and Stuart test for trend was carried out by splitting the points in Figure 5 into two halves and comparing the pairs. Since all of the scores in the upper half exceed their partners in the lower half, we can reject the null hypothesis that there is no trend and assume that a valid upward trend exists in performance as the depth of the disparities increases. This confirms that a functional relationship exists between disparity and performance and that our subjects saw stereoscopic depth at this very brief exposure.

It is noteworthy that even the 2 observers who did not do as well as the other 2 performed at very high levels for the paraboloid of rotation. Clearly, a high degree of stereoscopic competence can be demonstrated for selected stimuli for all the observers, even at the very brief exposures used in this study.

\section{DISCUSSION}

The major conclusion to be drawn from this work is that stereoscopic perception is not only possible, but, contrary to the current controversy, can be virtually perfect at very brief exposure durations if adequate convergence has been established so that good registration exists at the time of the brief stimulation. All of our observers were able to perform well, and 2 performed nearly perfectly in at least some of the daily sessions. Indeed, given the large depth differences between the closest and farthest dots of a stimulus form (as exemplified by the paraboloid of rotation), the other 2 observers also performed nearly perfectly. Whatever the causes of their stereoscopic recognition deficiencies, the performance of these 2 observers deteriorated only when the stimulus forms were relatively flat.

Stereoscopic recognition of nonplanar surfaces with brief exposures is, therefore, dependent upon the mag- nitude of the depth in the test stimulus. It was also shown to be highly linear as a function of this dimension (see Figure 5), with a curious dip at small disparities. Lehmkuhle and Fox (1980) also observed a similar nonmonotonicity, but at a larger disparity (at about $20^{\prime}$ disparity) and, curiously, in the opposite direction. A correlation coefficient calculation shows that the relationship between the pooled performance of all 4 observers and the range of disparities within each form in the stimulus set is $91.7 \%$ - a comfortably high value. Obviously, the range of stereoscopic disparities is a strong predictor of the results of this experiment.

Of course, if the stimuli had been masked by extraneous randomly positioned dots, or if the registration of the dichoptic images on corresponding retinal points had not been so carefully controlled by the viewing conditions of this experiment, the high levels of performance observed here could not have been produced. We believe that this latter factor-good registration by establishing solid fixation-accounts for the difference between these and previous results (e.g., Richards, 1977), which have suggested that stereopsis is poor at brief stimulus exposures unless monocular cues are present. The observers in the present study were provided with a stable fixation/convergence point for a full second before the stimulus presentation in order to establish correspondence between the two monocular views. In Richards's experiment, subjects were presented with a more complex preliminary display that appears not to have established adequate fixation disparity. The results presented here create substantial difficulties for any theory of stereopsis that requires eye movements. Our stimuli were so brief that there was no such possibility.

We conclude that any report of a stimulus duration threshold for the perception of stereoscopic depth is due to the fact that the observers were not provided with suf- 
ficient cues or preparatory time to establish adequate dichoptic registration. The estimate of $200 \mathrm{msec}-\mathrm{set}$ earlier as a temporal threshold for monocular-cue-free stereopsis-therefore, is probably a more realistic measure of the time that it takes to converge the eyes and establish the correspondence between the two images, rather than the minimum time that well-registered stimuli must be present to provide an adequate stimulus for stereoscopic depth perception. The results of the present study lead us to conclude that, as far as stereopsis is concerned, there is no minimum stimulus duration required for stereopsis, with or without monocular cues. Of course, the neural and perceptual response is prolonged by the impulse function of the entire visual system. However, this controversy has been focused on stimulus duration.

Finally, it should also be noted that the question we ask here concerning the stimulus duration is not the same as that concerning the amount of perceptual processing time required to form the stereoscopic impression after the stimuli have been registered. Both Julesz (1964) and Uttal, Fitzgerald, and Eskin (1975) have answered that question (approximately $50 \mathrm{msec}$ ) by using poststimulus masking with varying intervals between the stimulus and the mask. Obviously, the brief physical stimulus duration and the duration of the prolonged neural events that process the information carried by the stimulus may be quite different.

\section{REFERENCES}

FOLEY, J. M., \& RICHARDS, W. (1974). Improvement in stereoanomaly with practice. American Journal of Physiological Optics, 51, 935-938.

Groner, R., Groner, M. T., Muller, P., Bischof, W. F., \& Di LoLLo, V. (1993). On the confounding effects of phosphor persistence in oscilloscopic displays. Vision Research, 33, 913-918.

JONES, R. (1977). Anomalies of disparity detection in the human visual system. Journal of Physiology, 264, 621-640.
JuLESZ, B. (1960). Binocular depth perception of computer generated patterns. Bell System Technical Journal, 39, 1125-1162.

JuLESZ, B. (1964). Binocular depth perception with familiarity cues. Science, 145, 356-362.

LeHMKuHle, S., \& Fox, R. (1980). Effect of depth separation on metacontrast masking. Journal of Experimental Psychology: Human Perception \& Performance, 6, 605-621.

Newhouse, M., \& UtTaL, W. R. (1982). Distribution of stereoanomalies in the general population. Bulletin of the Psychonomic Society, 20, 48-50.

Patterson, R., \& Fox, R. (1984a). The effect of testing method on stereoanomaly. Vision Research, 24, 403-408.

PAtTerson, R., \& Fox, R. (1984b). Stereopsis during continuous head motion. Vision Research, 24, 2001-2003.

RICHARDS, W. (1970). Stereopsis and stereoblindness. Experimental Brain Research, 10, 380-388.

RICHARDS, W. (1971). Anomalous stereoscopic depth perception. Journal of the Optical Society of America, 61, 410-414.

RICHARDS, W. (1977). Stereopsis with and without monocular controls. Vision Research, 17, 967-969.

Staller, J. D., Lappin, J. S., \& Fox, R. (1980). Stimulus uncertainty does not impair stereopsis. Perception \& Psychophysics, 27, 361367.

Steinman, R. M., \& Collewijn, H. (1980). Binocular retinal image motion during active head rotation. Vision Research, 20, 415-429.

Steinman, R. M., Levinson, J. Z., Collewijn, H., \& Van der STEEN, J. (1983). Vision in the presence of known retinal image motion. Journal of the Optical Society of America, 73, 1856.

UTTAL, W. R. (1985). The detection of nonplanar surfaces in 3-D space. Hillsdale, $\mathrm{NJ}$ : Erlbaum.

UTTAL, W. R. (1987). The perception of dotted forms. Hillsdale, NJ: Erlbaum.

UtTal, W. R., Baruch, T., \& Allen, L. (1994). Dichoptic and physical information combination: A comparison. Manuscript submitted for publication.

Uttal, W. R., Davis, N. S., Welke, C., \& Kakarala, R. (1988). The reconstruction of static visual forms from sparse dotted samples. Perception \& Psychophysics, 43, 223-240.

UtTal, W. R., Fitzgerald, J., \& Eskin, T. E. (1975). Parameters of tachistoscopic stereopsis. Vision Research, 15, 705-712.

(Manuscript received March 9, 1994; revision accepted for publication May 23, 1994.) 